\title{
Development of Noise-Measuring System Based on the Wireless Sensor Network
}

\author{
Zhi-Kai Zhang, Jian Wu \\ Zhejiang Province Institute of Metrology, Hangzhou, China \\ E-mail:zzkhello520@163.com,15858268533@163.com
}

\begin{abstract}
In order to solve the measurement of noise in long term and real time on line way, the noise-measuring system based on the wireless sensor network is designed and developed. It can be used collected at the unattended measuring-points, and transmitted to the data center. After data processing, it will get the results of the noise. The experimental results show that the testing results of this system can reflect the real situation of the noise test area, and it can be widely used in some applications which need to control noise pollution or noise monitor in a period of time.
\end{abstract}

Keywords-wireless sensor network; noise-measuring system; measuring-points; noise monitor

\section{INTRODUCTION}

With the rapid development and expansion of the city, noise pollution is becoming more and more serious. As noise pollution will disturb daily life of residents, induce various diseases and cause hearing loss, the measuring, prevention and control of noise has been the research hotspot in many fields such as environment, traffic, architecture and acoustics[1]. At present, the most widely used method is still measuring the noise with noise-measuring instrument manually. However, when measuring the noise continuously in a period of time, testers are not allowed to leave, they should operate the instrument. Sometimes, in order to realize the statistics and evaluation of the noise data and the control of noise, testers will have to spend 24 hours or more to monitor the object for noise-measuring to obtain the real and complete noise data, which consumes lots of effort and time[2]. So the wireless on-line measurement of noise has become an urgent requirement for environment noise monitoring and noise testing workers, and with the development of Wireless Sensor Network, the wireless online measurement of noise is able to be realized [3-5].

Under this background, the noise-measuring system based on the wireless sensor network was designed and developed, which could not only realize the real time noise data collection, transmission and analysis, but also the real time display of the noise strength distribution.

\section{SYSTEM ARCHITECTURE}

\section{A. System General Design}

As shown in Fig. 1 that the noise-measuring system based on the wireless sensor network consists of three parts: perceptional layer for noise monitoring, transport layer for noise data, application layer for noise monitoring result.

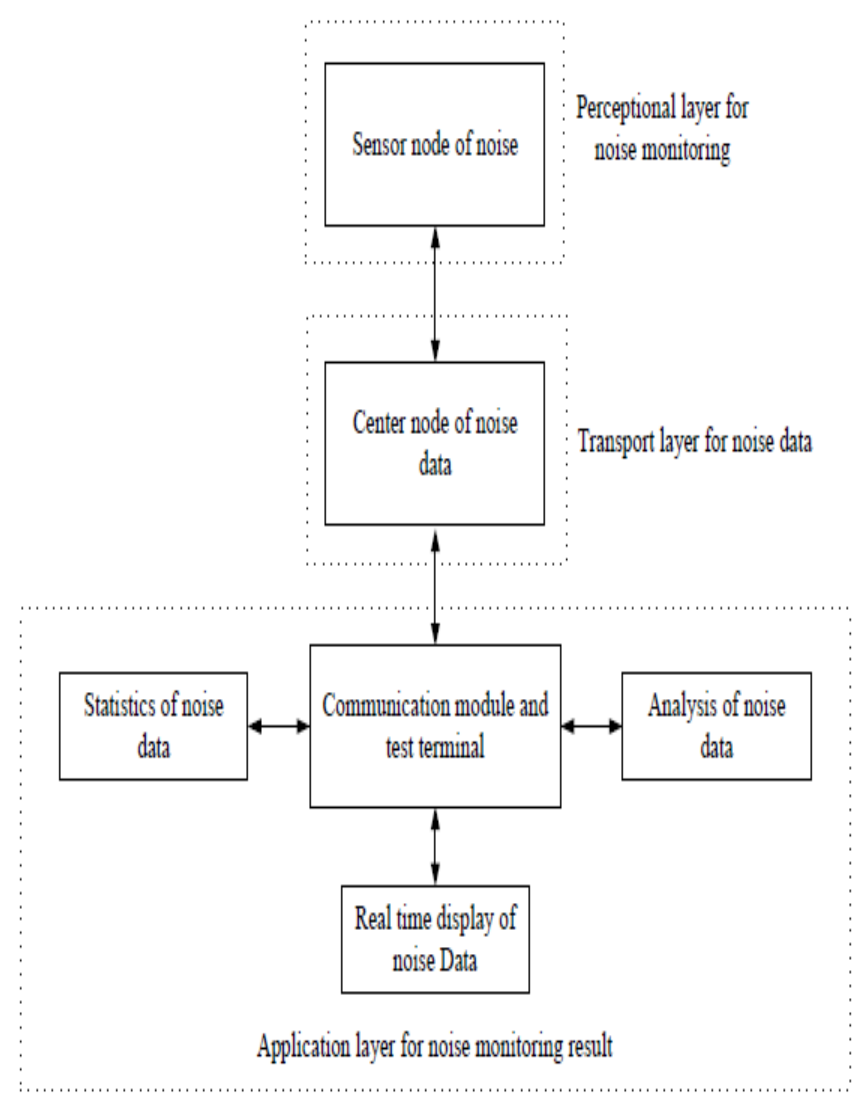

Figure 1. Schematic diagram of noise-measuring system.

- Perceptional layer for noise monitoring: mainly responsible for the real-time collection of noise, including the sensor module, power module, auxiliary module for monitoring (fixture, tripod, etc.)

- Transport layer for noise data: mainly responsible for transfer the data collected by noise sensor to the center node of data center.

- Application layer for noise monitoring result: mainly responsible for the statistics and analysis of noise data. It mainly consists of two parts: hardware and software, the hardware provides the storage space and software platform, and the software the provides the real time monitoring, data statistics, data analysis, and the display of monitoring result.

\section{B. Software and Hardware Design}

The noise-measuring system is made up of three modules: PC control module, wireless transmitting module, and noise sensor module. The sensor node is responsible for collecting 
the noise data in test area, converting analog signals into data signals, and transmitting the data to the data center node by multi hop mode. The data center node is used to connect the wireless sensor network, transmission network and control terminal[6]. During the experiment, the data will be transmitted to control terminal by means of wireless transmission method, the real time data acquisition, display and analysis will be shown on the control terminal by means of virtual instrument technology. To strengthen the reliability of wireless transmission network, the central node of data, which is on the basis of tree form distribution in the test area, is taken as the fundamental point. The wireless transmission protocol is Zigbee protocol, and the structure between nodes is net structure[7].

Thenoise-measuring sensor node is mainly responsible for collecting the noise data in test area, and transmitting the data to the data center node. As the Fig. 2 shows that the noise-measuring sensor node is consists of four modules: data acquisition module, data processing module, wireless data transmission module and power module.

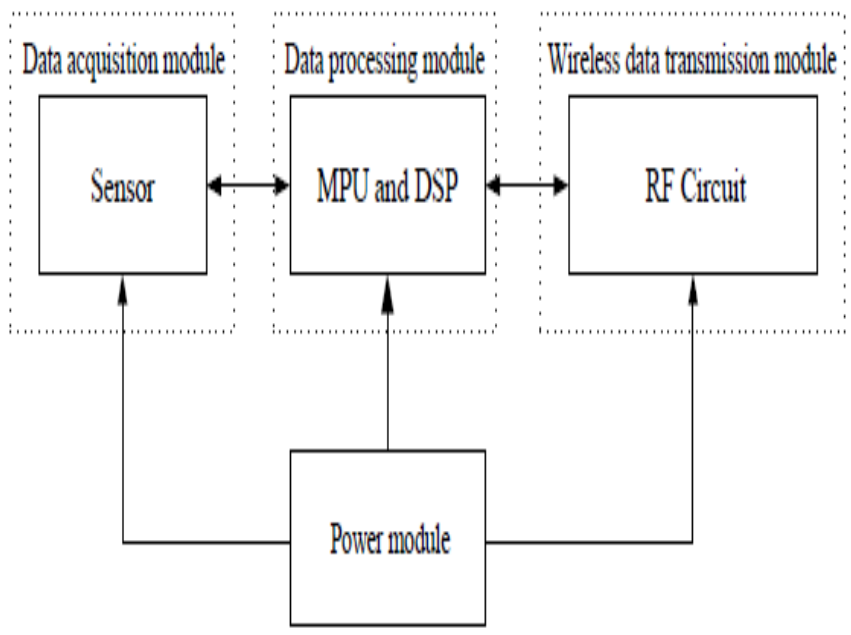

Figure 2. Schematic diagram of noise-measuring sensor node.

Data acquisition module is responsible for measuring the noise data in test area, data processing module is responsible for converting analog signals into data signals, wireless data transmission module is responsible for the communication and data transmission between nodes, and power module is responsible for providing the power for data acquisition module, data processing module and wireless data transmission module during the test.

Data center node is the noise data collection center based on Zigbee[8-9], which can realize the real-time communicate with multiple sensors. The data obtained on the test node will be transmitted to the data center node first, and then transmitted to the control terminal instantly. The real time communication between control terminal and data center node is based on the virtual instrument technology. VISA module can realize the data reading and writing, during the experiment, the tester can not only measure the real-time noise distribution in test area, but also process the data with the human computer interaction platform. The flowchart of the test program of control terminal is shown in Fig.3.

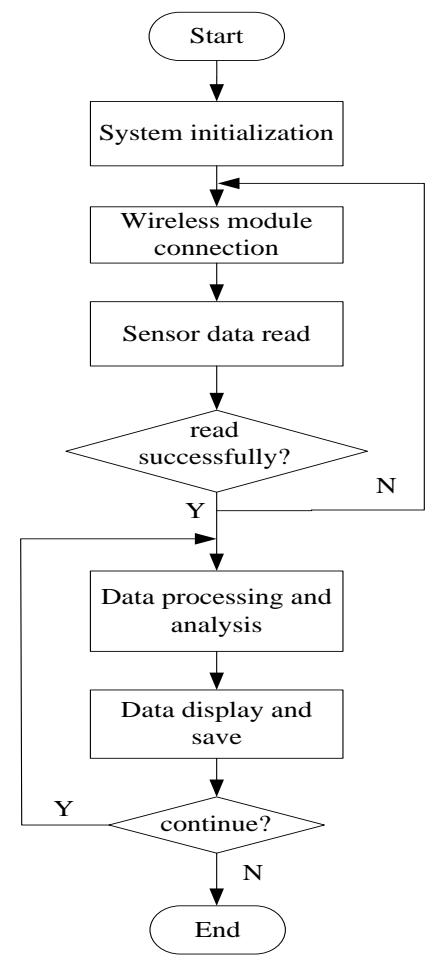

Figure 3. The flowchart of the test program of control terminal.

\section{APPLICATION OF THE SYSTEM}

The application of noise-measuring system based on the wireless sensor network is divided into three stages: prophase preparation, data collection of system and test data analysis.

\section{A. Prophase Preparation}

- Determination of test area

- The environmental test area is in scientific research unit, whose test area is 1 type according to the environmental quality standard for noise, the diurnal limit of noise is $55.0 \mathrm{~dB}[10]$.

- Preparation of test equipment

- The power supply of the noise-measuring system is battery, and the senor distance between the position of the sensors and the ground should be at least $1.2 \mathrm{~m}$ away.

- Noise test points layout

The test point is multipoint distribution, the distance between sensors and any other reflector should be at least $3.5 \mathrm{~m}$ away.

\section{B. Data collection of system}

During the experiment, every test point will transmit the noise data to the terminal through wireless sensor network for analyzing. The test time lasts for 5 days, after the data is processed, the system will select 500 sets data from each collection point to analysis.

\section{Test result analysis}

Equivalent continuous sound pressure level is used to reflect the noise variation with time, 


$$
L_{e q}=10 \lg \left[\frac{1}{T} \int_{0}^{T} 10^{0.1 L_{P A}} d t\right]
$$

Where: LPA is the Instantaneous sound pressure level.

$\mathrm{T}$ is the measured time.

The equivalent continuous sound pressure level can be obtained through calculation, as shown in Table 1.

\section{TABLE I. EQUIVALENT CONTINUOUS SOUND PRESSURE} LEVEL

\begin{tabular}{|c|c|c|c|c|c|}
\hline $\begin{array}{c}\text { Monitor } \\
\text { Point }\end{array}$ & $\begin{array}{c}\text { 1st Day } \\
(\mathrm{dB})\end{array}$ & $\begin{array}{c}\text { 2nd Day } \\
(\mathrm{dB})\end{array}$ & $\begin{array}{c}\text { 3rd Day } \\
(\mathrm{dB})\end{array}$ & $\begin{array}{c}\text { 4th Day } \\
(\mathrm{dB})\end{array}$ & $\begin{array}{c}\text { 5th Day } \\
(\mathrm{dB})\end{array}$ \\
\hline 1 & 70.1 & 67.7 & 53.8 & 58.4 & 65.3 \\
\hline 2 & 66.3 & 50.5 & 50.3 & 53.0 & 51.5 \\
\hline 3 & 52.5 & 49.6 & 53.0 & 49.4 & 55.4 \\
\hline 4 & 45.2 & 57.9 & 49.7 & 48.5 & 48.5 \\
\hline 5 & 68.0 & 48.5 & 53.8 & 49.6 & 43.5 \\
\hline 6 & 53.2 & 52.3 & 50.9 & 52.8 & 51.9 \\
\hline 7 & 42.4 & 42.3 & 41.5 & 41.4 & 41.1 \\
\hline 8 & 52.1 & 50.7 & 50.6 & 52.3 & 50.1 \\
\hline
\end{tabular}

From Table 1 of the noise distribution can be obtained, that the park management can take different noise control measures for different noise test area.

\section{CONCLUSION}

Using wireless sensor networks to achieve data acquisition is an effective way to test and analyze the noise in the future. The noise measuring system based on wireless sensor network can realize the continuous measurement of environmental noise, and has the characteristics of high efficiency, real-time analysis, real-time measurement and low energy consumption. What's more, compared with other noise-measuring system, the installation is relatively simple, the mobility is strong, this system can be widely used in urban environmental noise test or the need for a long time to monitor noise, which has great application value.

\section{ACKNOWLEDGMENT}

This research was supported by Zhejiang Bureau of Quality and Technical Supervision project 20150217. Prof. $\mathrm{Hu}$ Fei in Shanghai Jiaotong University is thanked for sharing her Software experience with the authors.

\section{REFERENCES}

[1] H.X. Li, J. Zhao, "Study on evaluation method of the regional ambient noise based on GIS tools," Remote Sensing Technology and Application, 2006, 21(4), pp. 363-367.

[2] H. Li, H. L.Xing, and Y. W. Li, "On-line auto monitoring system of environmental noise," Environmental Science and Managemeng, 2005, 30(4), pp. 101-102.

[3] X. H. Cao, J. H. Yang, and L. W. Chen, "Design of Distributed City Noise Monitoring System Based on Wireless Sensor Network," Sensors and Actuators, 2013, 26(8), pp. 1159-1162.

[4] X. F. Mao, M. Yang, and D. L. Mao, "Survey on wireless sensor network applications," Computer Applications and Software, 2008, 25(3), pp. 179-181.

[5] X. Q. Yin, Q. Y. Zhu, "Noise Monitoring System based on WiFi and Virtual Instrument," Computer Measurement and Control, 2015, 23(12), pp. 4002-4004.

[6] K. Xu. "Power measurement system based on Zigbee wireless sensor network," Metrology and Mesurement Technology, 2010,30(4), pp. $39-42$.

[7] Jangra, "Wireless sensor network(WSN):Architectural design issues and vhallenges," International Journal on Computer Science and Engineering, 2010, 2(9), pp. 3089-3094.

[8] Wang, H. L. Liu, and K. Xu, "Analysis and Energy Consumption of Physical Layer of Wirless Sensor Network under Differernt Channels," Computer Engineering and Applications, 2016, 52(12), pp. 112-116.

[9] J. Yick. "Wireless sensor network survey," Computer Network, 2008, 52(12), pp. 2292-2330.

[10] General administration of quality supervision, inspection and quarantine, "Environmental Quality Standard for Noise", GB 30962008, 2008. 